\title{
How to Differentiate Borderline Hepatic Nodules in Hepatocarcinogenesis: Emphasis on Imaging Diagnosis
}

\author{
Hyun Jeong Park Byung Ihn Choi Eun Sun Lee Sung Bin Park \\ Jong Beum Lee \\ Department of Radiology, Chung-Ang University Hospital, Chung-Ang University College of \\ Medicine, Seoul, Republic of Korea
}

\section{Keywords}

Hepatocarcinogenesis - Dysplastic nodule · Hepatocellular carcinoma - Imaging diagnosis ·

Borderline nodule

\begin{abstract}
Background: Rapid advances in liver imaging have improved the evaluation of hepatocarcinogenesis and early diagnosis and treatment of hepatocellular carcinoma (HCC). In this situation, detection of early-stage HCC in its development is important for the improvement of patient survival and optimal treatment strategies. Because early HCCs are considered precursors of progressed HCC, precise differentiation between a dysplastic nodule (DN), especially a high-grade DN, and early HCC is important. In clinical practice, these nodules are frequently called "borderline hepatic nodules." Summary: This article discusses radiological and pathological characteristics of these borderline hepatic nodules and offers an understanding of multistep hepatocarcinogenesis by focusing on the descriptions of the imaging changes in the progression of DN and early HCC. Detection and accurate diagnosis of borderline hepatic nodules are still a challenge with contrast enhanced ultrasonography, CT, and MRI with extracellular contrast agents. However, gadoxetic acid-enhanced MRI may be useful for improving the diagnosis of these borderline nodules. Key Messages: Since there is a net effect of incomplete neoangiogenesis and decreased portal venous flow in the early stage of hepatocarcinogenesis, borderline hepatic nodules commonly show iso- or hypovascularity. Therefore, precise differentiation of these nodules remains a challenging issue. In MRI using hepatobiliary contrast agents, signal intensity of HCCs on hepatobiliary phase (HBP) is regarded as a potential imaging biomarker. Borderline hepatic nodules are seen as nonhypervascular and hypointense nodules on the HBP, which is important for predicting tumor behavior and determining appropriate therapeutic strategies.




\section{Liver Cancer}

\section{Introduction}

Hepatocellular carcinoma (HCC) is the sixth most common tumor and the third most common cause of death in the world [1-4]. Approximately $80 \%$ of HCCs develop in cirrhotic liver caused by various long-standing hepatic injuries including chronic hepatitis B or C virus infections, alcohol, nonalcoholic steatohepatitis or autoimmune hepatitis [5]. The prognosis of patients with HCC depends largely on the stage at which the tumor is detected. Treatments with curative intentions such, as liver transplantation, surgical resection, or radiofrequency ablation, are applicable only in patients with early-stage HCC, and may provide much better survival than in those with advanced-stage HCC [6]. Therefore, detection of the early-stage HCC in its development is essential for the improvement of patient survival. However, precise diagnosis of the tumor is sometimes challenging not only by imaging techniques but also by histopathological examinations.

Meanwhile, long-standing chronic inflammations evoke genetic variations resulting in hepatocarcinogenesis [7]. In this condition, HCC frequently shows multistep hepatocarcinogenesis characterized by the sequence from dysplastic nodule (DN), early HCC to progressed HCC [8]. Because early HCCs are considered precursors of progressed HCC, the precise differentiation between DN (especially high-grade DN) and early HCC is important $[9,10]$. In clinical practice, these nodules are frequently called "borderline hepatic nodules." However, detection and precise differentiation of borderline hepatic nodules have been difficult and remain uncertain to date. The main reason for these issues is that the pathological features of these borderline nodules are very similar [11].

Rapid advances in liver imaging have improved the evaluation of hepatocarcinogenesis and the early diagnosis of HCC. These imaging technologies include contrast enhanced ultrasonography (CEUS), multidetector row CT, and high-quality magnetic resonance imaging (MRI) with tissue-specific contrast agents. However, there are still limitations to an accurate characterization and diagnosis of borderline hepatic nodules.

This article discusses radiological and pathological characteristics of these borderline hepatic nodules and offers insight into the multistep hepatocarcinogenesis by focusing on describing the imaging changes involved in the progression of DN and early HCC.

\section{Hepatocarcinogenesis: Pathological Changes}

Hepatocarcinogenesis is a multistep process characterized by the progression of successively more advanced precancerous, early cancerous, and progressed cancerous lesions [10, 12-15]. During multistep hepatocarcinogenesis, the most common terminology of the International Working Party of the World Congress of Gastroenterology (ICGHN) specified regenerating nodules (RNs), low-grade DNs (LGDNs), high-grade DNs (HGDNs), and HCC as the steps involved in the progression from RN to HCC [15-19].

Borderline hepatic nodules include precancerous lesions such as dysplastic foci and DNs, as well as early HCC [17]. Pathologically, dysplastic foci are defined as clusters of hepatocytes with precancerous features such as small cell change, measuring less than $1 \mathrm{~mm}$ in diameter [20]. Therefore, these small lesions are not detected by in vivo imaging, which remains a challenge for future investigation. DNs are precancerous hepatocellular lesions that contain dysplastic features without histological evidence for malignancy. They are subcategorized into LGDN and HGDN depending on the presence of cytological and architectural atypia [15, $16,21,22]$. DNs are about $1-1.5 \mathrm{~cm}$ in diameter and are differentiated from the surrounding parenchyma by size, color, and texture [10]. The cells of HGDNs show cellular atypia; however, the atypia is insufficient for establishing a diagnosis of HCC. Clinically, HGDNs are considered 


\section{Liver Cancer}

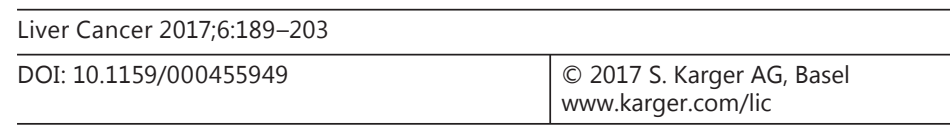

Park et al.: How to Differentiate Borderline Hepatic Nodules in Hepatocarcinogenesis: Emphasis on Imaging Diagnosis

precursors of HCC with a high risk of transformation [10,23]. Histologically, DNs may contain more copper and/or iron than background liver [24]. LGDNs and most HGDNs have relatively preserved arterial blood supply; therefore, they usually show isoattenuation or isointensity with respect to the liver on the arterial phase of the CT image or MRI. Therefore, they are not well visualized by CT or MRI.

Recently, an international consensus has been reached regarding the histological features of an early HCC, which is an early stage of HCC development with indistinct margins ("HCC of vaguely nodular type" or "small and early HCC") in contrast to small and progressed HCC with distinct margins ("HCC of distinctly nodular type" or "small and progressed HCC") $[16,17]$. Small HCC is arbitrarily defined as carcinomas measuring $<2 \mathrm{~cm}$ in diameter. Early HCCs are similar to "carcinoma in situ" of other organs, and show replacing growth into the surrounding parenchyma [10]. The key pathological feature of early HCCs which is helpful for differentiating them from HGDNs is stromal invasion, which is defined as the infiltration of tumor cells into the fibrous tissue surrounding the portal tracts and replacing growth. They are considered precursors of progressed HCCs, not showing vascular invasion or intrahepatic metastases [16]. It is suggested that detection of heat shock protein-70, glutamine synthetase, and glypican-3 by immunohistochemical stains is a useful adjunct in distinguishing early HCC from DNs $[25,26]$. In contrast, "small and progressed HCCs" or "small distinctly nodular HCCs" show expansive growth, frequently form a tumor capsule, and are associated with vascular invasion, as well as intrahepatic metastases [10]. Therefore, the precise diagnosis of early HCC and adequate management may prolong patient survival. However, pathological features of an early HCC are quite similar to those of an HGDN [11]. Besides, it is still recognized that there are considerable differences between Western and Eastern pathologists in the pathological diagnosis of borderline nodules, including DN and early HCC causing difficulty in research and clinical management of these lesions. Many early HCCs diagnosed by Japanese pathologists tend to be diagnosed as HGDNs by Western pathologists. By contrast, most HGDNs diagnosed by Western pathologists tend to be diagnosed as well-differentiated HCC by the Japanese [27].

\section{Key Alterations during Hepatocarcinogenesis}

During the hepatocarcinogenesis, Kupffer cell density, hepatocyte function, portal tracts, and organic anionic transporting polypeptide (OATP) expression simultaneously and gradually decrease, while sinusoidal capillarization and recruitment of unpaired arterioles develop. In addition, venous drainage from hepatic veins diverts to portal veins. Intranodular fat content usually increases in early hepatocarcinogenesis, but regresses in progressed HCC (Fig. 1) [28, 29].

\section{Angiogenesis and Venous Drainage}

Neoangiogenesis is characterized by the development of nontriadal or unpaired arteries and sinusoidal capillarization detected by immunohistochemical stain for CD34. Unpaired arteries are isolated arteries unaccompanied by portal veins or bile ducts. The vascular supply of DNs is derived from unpaired arteries and portal tracts, which are induced by growth factor produced by lesional cells [30-32]. It gradually advances according to the development of multistep hepatocarcinogenesis. In small and early HCCs, neoangiogenesis is not fully developed yet. In early HCCs, the zone of sinusoidal capillarization is sparse, and the number of unpaired arteries is small [30,31]. Glutamine synthetase (a target protein of $\beta$-catenin), 
Park et al.: How to Differentiate Borderline Hepatic Nodules in Hepatocarcinogenesis: Emphasis on Imaging Diagnosis

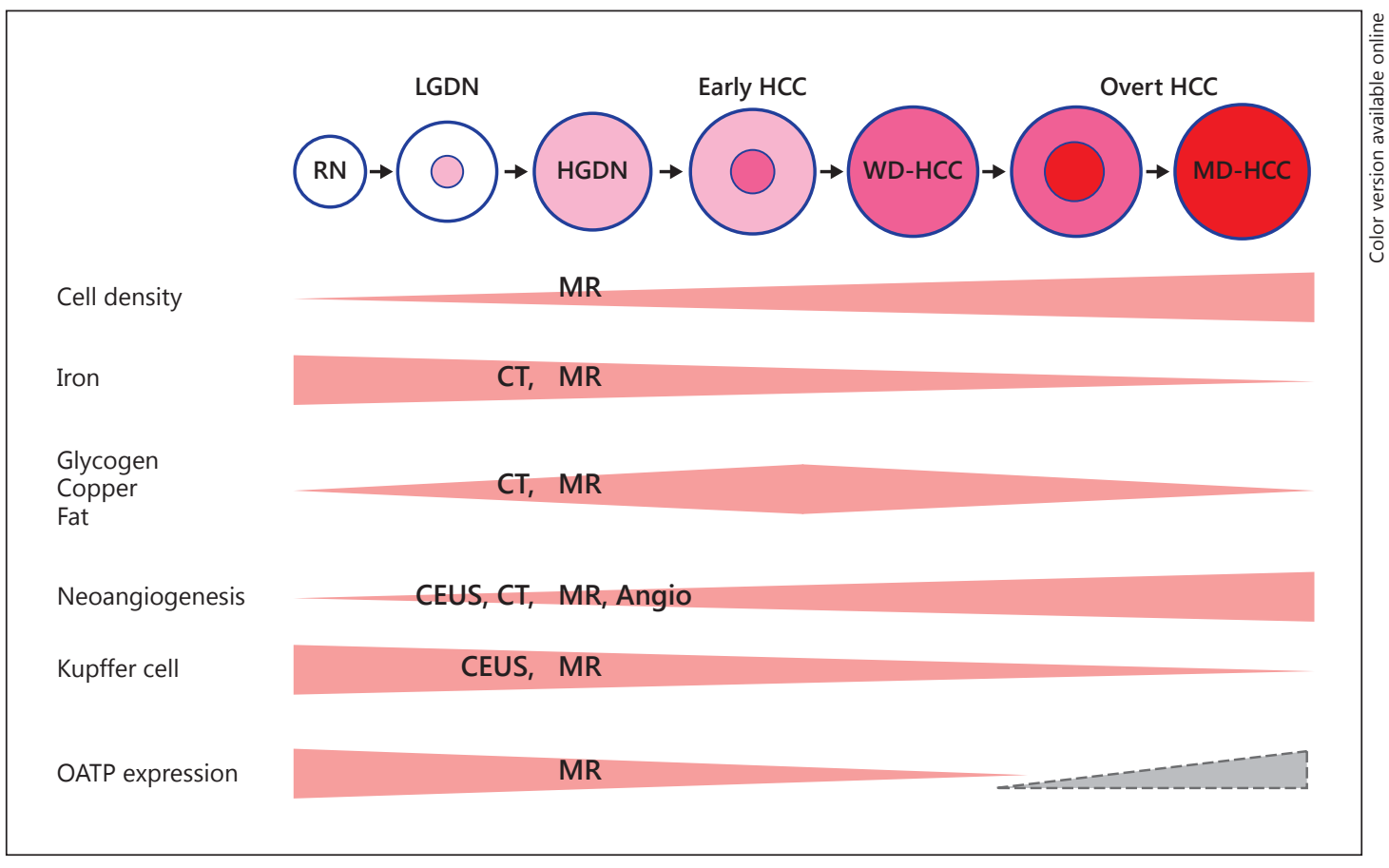

Fig. 1. Cellular and histopathologic changes and tumor visualization on imaging modalities during hepatocarcinogenesis. RN, regenerating nodule; LGDN, low-grade dysplastic nodule; HGDN, high-grade dysplastic nodule; HCC, hepatocellular carcinoma; WD-HCC, well-differentiated HCC; MD-HCC, moderate-differentiated HCC; CEUS, contrast-enhanced US; Angio, angiography; OATP, organic anionic transporting polypeptides. Dotted line in OATP expression indicates paradoxically overexpressed OATP in overt HCCs. Modified figure based on figures from Drs. Kojiro, Kudo, Matsui, Bartolozzi, Lim.

glypican-3 (a membrane proteoglycan), and heat shock protein-70 (a chaperone stress protein) have recently been reported for the early detection of early HCCs. Tissue detection of at least 2 immunomarkers can differentiate malignancy in an equivocal hepatocellular nodule $[33,34]$. In parallel with these alterations, nontumoral hepatic arteries and portal veins (portal tracts) gradually diminish [29]. During hepatocarcinogenesis, venous drainage diverts from hepatic veins in cases with RNs, DNs, and early HCCs through sinusoids to portal veins in cases with progressed HCCs with fibrous capsules [35, 36].

\section{Fat and Iron Contents}

In the early stage of hepatocarcinogenesis, LGDNs, HGDNs, and early HCCs show increased fat accumulation in the hepatocytes when compared to that of the background liver [37]. The incidence increases from LGDN to HGDN and early HCC. Forty percent of early HCCs manifest diffuse fat accumulation [38]. The mechanism of fat accumulation in early hepatocarcinogenesis is presumed to be due to the deficient development of unpaired arteries and reduction of portal venous and nontumoral arterial flow, which induces intracellular fat accumulation in an ischemic/hypoxic environment [38]. After the development of unpaired arteries in progressed HCCs, the steatosis regresses [39]. Iron accumulation occurs preferentially in LGDNs and in some HGDNs. With further de-differentiation, most HGDNs, early HCCs, and progressed HCCs become iron free [40]. 


\section{Liver Cancer}

\section{OATP Transporters}

Several recent studies demonstrated that the expression of OATP, which are transporters of bile salts, diminishes during hepatocarcinogenesis: OATP expression levels are high in RNs and LGDNs and lower in many HGDNs, early HCCs, and progressed HCCs [28, 41]. During hepatocarcinogenesis, OATP8 expression level decreases prior to complete neoangiogenesis, elevation of arterial flow, and reduction of portal venous flow, which are important associations for imaging-based detection of HCC using hepatobiliary contrast agents [41, 42]. However, rarely, some well-differentiated HCCs and about 5-12\% of moderately differentiated HCCs paradoxically overexpress OATP (especially OATP8), which has been speculated to reflect a genetic alteration or different cellular origin during hepatocarcinogenesis [41].

\section{Imaging-Based Characterization of Borderline Hepatic Nodules}

\section{Ultrasonography}

To achieve detection and accurate characterization of borderline hepatic nodules in cirrhotic livers, application of state-of-the-art equipment and protocols is essential. Ultrasonography (US) has been used as a screening tool for HCC surveillance due to its safety, availability, and cost effectiveness. However, detection and sonographic features of HCC are nonspecific and diverse. With the background nodularity of cirrhotic liver, identification of borderline hepatic nodules is challenging because of its ill-defined border and small size. On gray-scale US, DNs manifest either as hypo-, iso-, or hyperechoic, which is similar to RNs or small HCCs [43]. Hyperechogenicity may be associated with fat content, while hypoechogenicity may reflect the internal homogeneity of DN (Fig. 2).

Currently, imaging assessment of the blood supply in these hepatic nodules is the most important parameter for characterization of diverse cirrhosis-associated nodules due to sequential angiogenesis and hemodynamic changes during hepatocarcinogenesis [44]. In Europe, Canada, and Asia, CEUS has been used as the third imaging modality, after CT and MRI, to detect and characterize various focal hepatic nodules, especially HCCs. Microbubblebased contrast agents are presently used and can be safely used in patients with renal failure since there is no renal excretion of the contrast agent [45]. On CEUS, DNs typically show isoor hypovascularity on arterial phase and isoechogenicity on subsequent phases $[46,47]$. In transient phases prior to the development of unpaired arteries, DNs frequently appear hypovascular on arterial phase because of the reduction of arterial and portal supplies [29]. Meanwhile, early HCC or well-differentiated HCC with a vaguely nodular appearance has variable degrees of arterial and portal venous supplies. In HCCs with nodule in nodule type, focal nodular hypervascularity within the hypovascular DNs on arterial phase is a valuable finding on imaging studies for the characterization of early HCC [47]. In cases where early HCCs have arterial hypovascularity, histologically fewer unpaired arteries are demonstrated [30, 31]. Jang et al. [48] and Takahashi et al. [49] reported that 39\% (9/23) and 41\% (22/54) of welldifferentiated HCCs demonstrated no hypervascularity on CEUS. Therefore, the differential diagnosis of borderline hepatic nodules including HGDNs and early HCCs is frequently challenging in clinical practice.

CEUS is a recent advancement that uses a liver-specific contrast agent (Sonazoid; DaiichiSankyo, Tokyo, Japan) that is taken up by Kupffer cells on the late phase. Intravenously injected perflubutane contrast agent reaches the sites of a Kupffer defect allowing for an easier and more definitive detection of malignancy. The Kupffer defect is assessed in the Kupffer phase 6-10 min after arterial enhancement of the target nodule. Some investigators have tried to differentiate borderline hepatic nodules using a contrast agent $[47,50,51]$. 


\section{Liver Cancer}
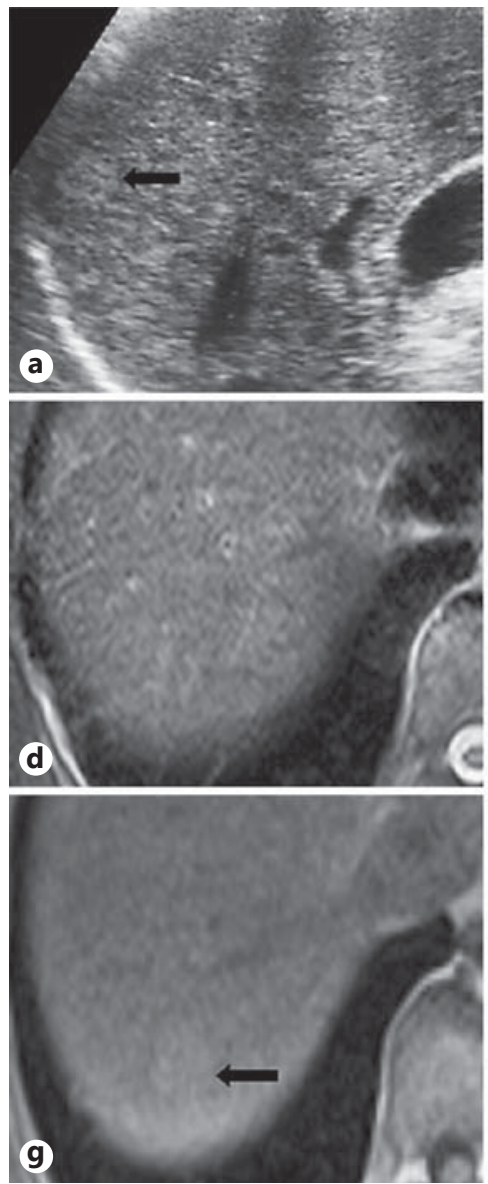

\begin{tabular}{l|l}
\hline Liver Cancer 2017;6:189-203 \\
\hline DOI: 10.1159/000455949 & $\begin{array}{l}\text { @ 2017 S. Karger AG, Basel } \\
\text { www.karger.com/lic }\end{array}$ \\
\hline
\end{tabular}

Park et al.: How to Differentiate Borderline Hepatic Nodules in Hepatocarcinogenesis: Emphasis on Imaging Diagnosis
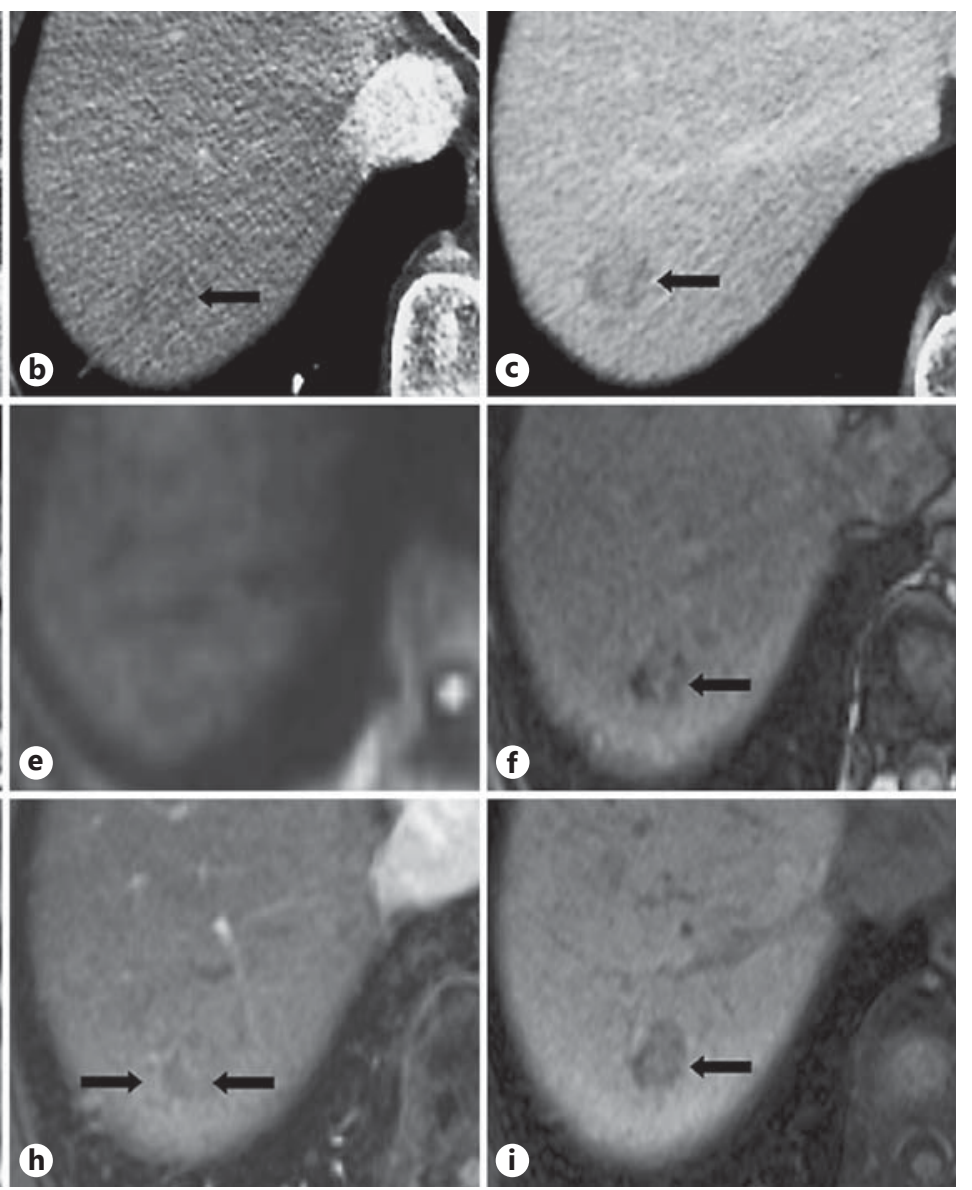

Fig. 2. Biopsy-confirmed high-grade dysplastic nodule in a 56-year-old woman with chronic hepatitis B virus infection. Transverse gray-scale ultrasound image (a) shows a 1.7-cm hyperechoic nodule (arrow) in segment 7 of the liver. On hepatic arterial phase (b), and 3-min delayed phase (c) CT images, the nodule (arrows) demonstrates persistent hypoattenuation. On Gd-EOB-DTPA-enhanced MRI, the nodule shows isointensity on the T2-weighted (d) and diffusion-weighted (b value $=800 \mathrm{~s} / \mathrm{mm}^{2}$ ) (e) images, and signal drop on the opposed-phase image (f) compared to the in-phase image (g). These findings are indicative of intratumoral fat. The nodule (arrows) reveals hypoenhancement on the arterial phase (h) and hypointensity on the 20min hepatobiliary phase (i). Courtesy of Ijin Joo from Seoul National University Hospital.

According to these studies, although no DNs showed hypoechogenicity in the Kupffer phase, 64-91\% of well-differentiated HCCs showed almost complete isoechogenicity. Therefore, the role of CEUS with perflubutane is limited for the differentiation of borderline hepatic nodules.

\section{Computed Tomography}

Currently, all major HCC guidelines recommend using multiphasic CT scanning, which should include late hepatic arterial, portal venous, and delayed-phase (about 3-5 min) acquisitions, with extracellular contrast agents as a first-line imaging modality for the diagnosis of HCCs [6, 52-54]. Most DNs show iso- or hypoattenuation on arterial, portal venous, and delayed-phase images because of the relatively preserved hepatic arterial flow (Fig. 2) [43, 55]. However, hepatic arterial flow in some HGDNs increases owing to neoangiogenesis resulting in the potential misdiagnosis of a hypervascular HCC [56]. These various imaging features are similar to early HCCs. It is reported that about $40 \%$ of HCCs lack the arterial- 


\section{Liver Cancer}

\begin{tabular}{l|l}
\hline Liver Cancer 2017;6:189-203 \\
\hline DOI: 10.1159/000455949 & $\begin{array}{l}\text { @ 2017 S. Karger AG, Basel } \\
\text { www.karger.com/lic }\end{array}$ \\
\hline
\end{tabular}

Park et al.: How to Differentiate Borderline Hepatic Nodules in Hepatocarcinogenesis: Emphasis on Imaging Diagnosis
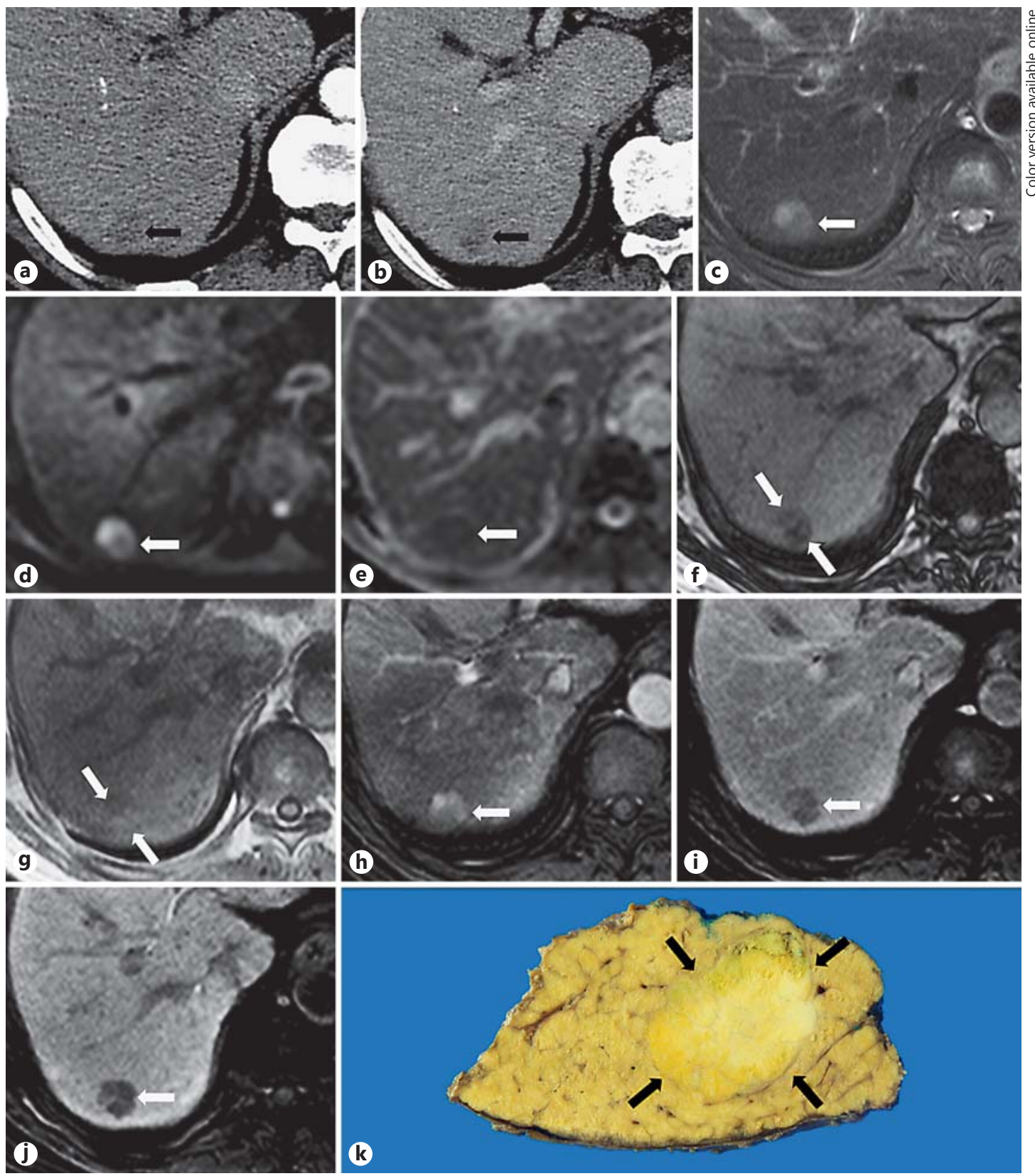

Fig. 3. Surgically confirmed early hepatocellular carcinoma (well-differentiated HCC with vaguely nodular type) in a 62-year-old man with hepatitis B virus-related cirrhosis. On CT images obtained after contrast injection during hepatic arterial (a) and 3-min delayed (b) phases, a 2-cm subtly hypoattenuated nodule (arrows) is noted in segment 6 of the liver. On Gd-EOB-DTPA-enhanced MRI, the nodule (arrows) shows hyperintensity on the T2-weighted image (c) and restricted diffusion on the diffusion-weighted image (b value = $800 \mathrm{~s} / \mathrm{mm}^{2}$ ) (d) and ADC map (e). On the opposed-phase image (f), foci (arrows) of decreased signal intensity are seen compared to the in-phase image (g), indicative of intratumoral fat. The nodule (arrows) demonstrates arterial hyperenhancement (h) and hypointensity on the 3-min delayed phase (i) and 20-min hepatobiliary phase (j) images. $\mathbf{k}$ Gross pathologic specimen reveals a vaguely nodular, yellowish tumor (arrows) without hemorrhage or necrosis. 
Park et al.: How to Differentiate Borderline Hepatic Nodules in Hepatocarcinogenesis: Emphasis on Imaging Diagnosis

phase hyperenhancement, which includes most early HCCs (Fig. 3) [57, 58]. This can lead to a misdiagnosis, using extracellular contrast agents, of nondefinitive HCC. Lee et al. [59] reported that only 7 of 16 histologically confirmed early HCCs were detected by preoperative CT scan and 4 HCCs demonstrated hypoenhancement. Similar results were reported in a study by Takayasu et al. [60]. Nearly half $(46 \%, 17 / 37)$ of the histologically proven early HCCs were not detected by CT scanning due to isoattenuation with respect to the background liver on unenhanced, arterial, and delayed enhanced CT scans. Furthermore, 95\% (20/22) of HCCs among the detected HCCs on CT revealed hypoenhancement. On dynamic contrast-enhanced CT, most early HCCs also did not show arterial-phase hyperenhancement because of preservation of the portal venous flow [60]. Therefore, although per-lesion sensitivity in CT is variable for borderline hepatic nodules in a cirrhotic liver, CT commonly shows low perlesion sensitivity. CT with extracellular contrast agents is an unreliable technique for differentiation of these nodules because of their usual features with iso- or hypoattenuation on all postenhanced CT images [61].

\section{Magnetic Resonance Imaging}

Techniques

Currently, because MRI provides better soft-tissue contrast and information of tissue components, it seems to be more useful than other modalities for the detection and characterization of borderline hepatic nodules and progressed HCCs in the face of liver cirrhosis [62]. Pathological characteristics of an increased cellular density, arterioportal imbalance, and decreased OATP expression during the hepatocarcinogenesis could be evaluated by various MRI techniques. This includes T1-weighted imaging including chemical shift imaging, T2-weighted imaging, dynamic enhanced MRI with gadolinium-based extracellular contrast agents, liver-specific contrast agents, and diffusion-weighted imaging (DWI) [62].

Precontrast T1- and T2-weighted imaging enables the identification of different tissue components both in the normal parenchyma and in a focal hepatic nodule. These components include the existence of fibrotic changes, water and fat contents, the entity of vascularization, and metabolites [63]. Arterioportal imbalance caused by increased arterial neovascularization and decreased portal venous flow is most frequently assessed by multiphasic dynamicenhanced MRI. This protocol includes at least 3 dynamic phases: the late hepatic arterial, portal venous (50 70 s after contrast injection), and delayed ( $2 \sim 3 \mathrm{~min}$ ) phases. Three types of MR contrast agents are available for the evaluation of cirrhosis-associated nodules including gadolinium-based extracellular contrast agents, superparamagnetic iron oxide (SPIO) contrast agents, and hepatobiliary contrast agents. Gadolinium-based extracellular contrast agents contain low-molecular-weight gadolinium chelates that cause T1-shortening and are useful for the evaluation of tissue vascularity [24]. SPIO and hepatobiliary contrast agents are liver-specific contrast agents. SPIO agents show uptake in the reticuloendothelial system (RES; so-called Kupffer cells), and the hepatobiliary agents show uptake in hepatocytes and demonstrate biliary excretion [9]. Clinically used hepatobiliary agents include gadobenate dimeglumine (MultiHance; Bracco Imaging, Milan, Italy) or gadoxetate disodium (Gd-EOBDTPA, gadoxetic acid disodium, Primovist; Bayer Schering Pharma, Germany). Recently, Gd-EOB-DTPA has been increasingly used to assess hepatic focal lesions including HCCs. It has dual properties that limit its distribution to the extracellular space. About $50 \%$ of the injection dose is actively taken up by hepatocytes via OATP8 and excreted into the bile duct via the multidrug resistance protein 2 . The other $50 \%$ is removed by renal excretion [64]. Therefore, Gd-EOB-DTPA-enhanced MRI can provide not only information about the vascular phases, but also additional information regarding the hepatobiliary phase (HBP). Dynamicenhanced images obtained with gadobenate dimeglumine have features similar to those obtained with other extracellular contrast agents. However, images obtained with Gd-EOB- 


\section{Liver

Park et al.: How to Differentiate Borderline Hepatic Nodules in Hepatocarcinogenesis: Emphasis on Imaging Diagnosis

DTPA are somewhat different since significant uptake of hepatocytes has occurred by the end of the portal venous phase. Although SPIO contrast agents are beneficial for distinguishing between benign hepatocellular nodules and progressed HCC based on different degrees of iron uptake, they are no longer available in many countries.

DWI as a functional imaging technique is now used widely as the standard imaging sequence of a liver MRI. DWI provides information on cellular architecture on a micrometer scale, and can evaluate the cellular density of hepatocellular nodules [62,65]. The degree of diffusion in water molecules per unit time is displayed using the apparent diffusion coefficient (ADC). Restricted diffusion means that the signal intensity is higher than the surrounding liver on DWI obtained with an intermediate to high range of $b$ values $\left(400-800 \mathrm{~s} / \mathrm{mm}^{2}\right)$. The ADC value of the nodule should be comparable to or lower than that of the surrounding liver by visual assessment.

Characterization of Borderline Hepatic Nodules Using MRI

The diagnostic performance for the diagnosis of HCCs is slightly better in dynamic MRI using extracellular contrast agents than multiphasic CT in several single-center comparative studies [66, 67], but the differences are small. Nonsiderotic DNs (nodules that are not ironrich) characteristically show hyperintensity on T1-weighted images and iso- or hypointensity on T2-weighted images [24,68]. Although the reason for these signal intensity characteristics is not well understood, the presence of paramagnetic materials or glycogen may contribute to T1 hyperintensity [69]. In contrast, siderotic or iron-rich nodules usually appear hypointense on T1-weighted images and moderately to markedly hypointense on T2-weighted and T2*-weighted images. However, depending on the iron concentration, these nodules may appear slightly hyperintense on T1-weighted images [40]. On MRI, early HCCs show either iso- or hyperintensity on T1-weighted images and typically are isointense on T2-weighted images [70], which suggests that an accurate diagnosis of borderline hepatic nodules using conventional MRI is difficult. Meanwhile, some DNs, especially HGDNs, contain higher intracellular fat than the background liver [71]. The nodules manifest as hyperintensity on T1-weighted in-phase images and have a signal drop on out-of-phase images (Fig. 2) [72]. However, since intracellular steatosis is more frequently identified in early HCC than in DNs and a small number of progressed HCCs, the presence of intracellular fat is not a characteristic feature of DNs [73]. Therefore, based on signal intensity characteristics on nonenhanced MRI alone, differentiation between DNs and HCCs is challenging because of the similar signal intensities on nonenhanced T1- and T2-weighted images [68].

With regard to the diagnosis of HCC, DWI has shown additional value to gadoliniumenhanced MRI by incrementally increasing the detection rate of HCC (Fig. 3) [74, 75]. The presence of restricted diffusion favors the diagnosis of malignancy and helps differentiate HCCs from DNs because restrictive diffusion reflects tissue hypercellularity [65]. However, considerable HCCs, particularly those with well-differentiated components, show no or minimal restricted diffusion [76].

According to current reports, Gd-EOB-DTPA-enhanced MRI enables the accurate detection and characterization of HCCs and has demonstrated an increased sensitivity for the detection of HCCs. In addition, Gd-EOB-DTPA-enhanced MRI has been reported to have a superior performance for detecting small HCCs compared to CT or MRI with extracellular contrast agents [77, 78]. Ichikawa et al. [79] reported that early HCCs were more frequently identified on HBP in Gd-EOB-DTPA-enhanced MRI (97\%) than on other imaging modalities including multiphasic CT, unenhanced MRI, and CT angiography (57-73\%). Since early HCCs show incomplete neoangiogenesis, isoattenuation on vascular phases is frequently seen in approximately $44 \%$ of early HCCs on multiphasic CT images [59]. However, because OATP8 expression decreases during hepatocarcinogenesis prior to complete neoangiogenesis and 
Park et al.: How to Differentiate Borderline Hepatic Nodules in Hepatocarcinogenesis: Emphasis on Imaging Diagnosis

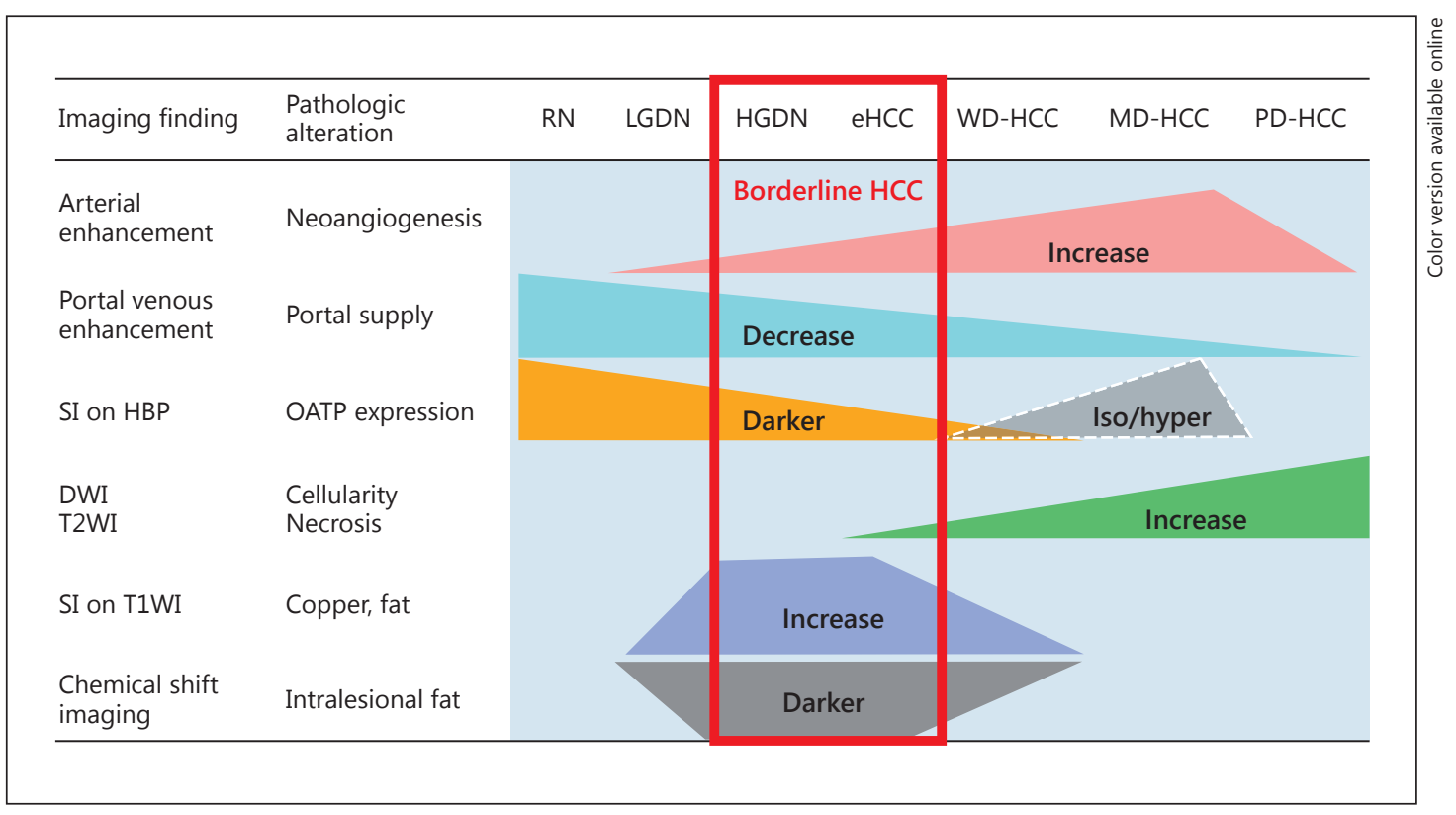

Fig. 4. Imaging features and pathologic alteration during hepatocarcinogenesis.

increased arterial flow, borderline hepatic nodules can be seen as nonhypervascular and hypointense nodules on HBP [80-82]. On Gd-EOB-DTPA-enhanced MRI, a considerable number of early HCCs, and some HGDNs, are hypointense on HBP [78] due to underexpression of OATP (Fig. 2 and 3). In contrast, most RNs, LGDNs, some HGDNs, and only a minority of HCCs show iso- or hyperintensity because of preserved OATP expression [83]. Therefore, precise differentiation of borderline hepatic nodules in cirrhotic liver using Gd-EOB-DTPA-enhanced MRI is a challenging issue. Recently, increasing interest has focused on the signal intensity of HCCs on HBP images as a potential imaging biomarker [84, 85]. Hyperintense HCCs on HBP have been reported to have histological features related to favorable outcomes more frequently than hypointense HCCs. In addition, nonhypervascular and hypointense nodules on HBP have gained growing interest due to the ability of borderline hepatic nodules to progress onto the subsequent sequences of hepatocarcinogenesis. According to recent studies, this development is not uncommon since 13.1-37.6\% of nonhypervascular and hypointense borderline nodules larger than $1 \mathrm{~cm}$ on HBP developed into hypervascular HCCs during the 12-month follow-up [86, 87]. Furthermore, approximately $30 \%$ of new hypervascular HCCs manifest as nonhypervascular and hypointense nodules on HBP before arterial hypervascularity develops on serial follow-ups with Gd-EOB-DTPAenhanced MRI [88]. Meanwhile, Ohama et al. [51] reported that 31 of 33 hypovascular welldifferentiated HCCs showed hypointensity on the HBP of Gd-EOB-DTPA-enhanced MRI, whereas only 3 of 33 hypovascular well-differentiated HCCs were hypoechoic on the Kupffer phase images on the Sonazoid-enhanced US. This study suggests that uptake of Sonazoid starts decreasing later than that of Gd-EOB-DTPA in stepwise hepatocarcinogenesis. Therefore, hypointensity on HBP images precedes hypoechogenicity on the Kupffer phase images, and the identification of these borderline hepatic nodules on HBP images is critical for predicting tumor behavior and deciding if the proper management of the lesions is treatment or expedited follow-up. 


\section{Liver Cancer}

\begin{tabular}{l|l}
\hline \multicolumn{2}{l}{ Liver Cancer 2017;6:189-203 } \\
\hline DOI: 10.1159/000455949 & $\begin{array}{l}\text { C 2017 S. Karger AG, Basel } \\
\text { www.karger.com/lic }\end{array}$ \\
\hline
\end{tabular}

Park et al.: How to Differentiate Borderline Hepatic Nodules in Hepatocarcinogenesis: Emphasis on Imaging Diagnosis

\section{Summary}

Imaging features and pathological alterations during hepatocarcinogenesis are summarized in Figure 4. During hepatocarcinogenesis, progressively increased unpaired arteries and diminished portal supply (contained portal veins and nontumoral hepatic arteries) lead to the hallmark diagnostic features of HCCs as hyperenhancement on arterial phase and the washout appearance on portal venous or delayed-phase images. Because of incomplete neoangiogenesis and decreased total blood flow, HGDNs and early HCCs are usually hypovascular relative to the background liver on arterial and portal venous phases. Intracellular fat and copper concentration are higher in HGDNs and early HCCS than in background liver and overt HCCs. The higher concentration causes a hyperintense change on a T1-weighted image. Intracellular steatosis is more frequently identified in early HCC and DNs, and it is identified as a hyperintensity on T1-weighted in-phase images and a signal drop on out-of-phase images. Typical overt HCCs show mild-moderate hyperintensity on T2-weighted image and restricted diffusion. Some borderline hepatic nodules also manifest these features. The level of OATP expression progressively decreases, and a considerable number of early HCCs and some HGDNs are hypointense on HBP. Therefore, due to similar and overlapping imaging features between HGDNs and early HCCs, accurate diagnosis of these borderline hepatic nodules is still challenging. However, a nonhypervascular and hypointense nodule on HBP is a strong predictor of premalignancy or malignancy, and this imaging feature can be identified prior to neoangiogenesis and progression to overt HCC. Hence, the identification of these borderline nodules for progression to overt HCCs on the basis of the imaging features is critical for determining the proper management strategy for these patients.

\section{Conclusions}

Angiogenic and functional assessments are currently the mainstay of the diagnosis of hepatocarcinogenesis. Correlation of functional imaging and molecular pathology is possible with new contrast agents. Detection and precise differentiation of borderline hepatic nodules such as HGDNs and early HCCs remain challenging issues. Multiparametric evaluation of tumor morphology, vascularity, cellularity, OATP function, and Kupffer cell function using functional imaging modalities with new molecular contrast agents may be useful for the characterization of these hepatic nodules. Diagnostic refinements can be achieved by using recent biomarkers, including imaging (DWI, Gd-EOB-DTPA-enhanced MRI) and pathological (molecular markers) biomarkers.

\section{Disclosure Statement}

The authors declare no conflicts of interest.

\section{References}

1 Ferlay J, Shin HR, Bray F, Forman D, Mathers C, Parkin DM: Estimates of worldwide burden of cancer in 2008: GLOBOCAN 2008. Int J Cancer 2010;127:2893-2917.

2 McGlynn KA, Tsao L, Hsing AW, Devesa SS, Fraumeni JF Jr: International trends and patterns of primary liver cancer. Int J Cancer 2001;94:290-296.

3 Parkin DM, Bray F, Ferlay J, Pisani P: Estimating the world cancer burden: Globocan 2000. Int J Cancer 2001; 94:153-156.

4 El-Serag HB: Hepatocellular carcinoma. N Engl J Med 2011;365:1118-1127. 
5 McGlynn KA, London WT: Epidemiology and natural history of hepatocellular carcinoma. Best Pract Res Clin Gastroenterol 2005;19:3-23.

6 Bruix J, Sherman M; American Association for the Study of Liver Diseases: Management of hepatocellular carcinoma: an update. Hepatology 2011;53:1020-1022.

7 Wurmbach E, Chen YB, Khitrov G, Zhang W, Roayaie S, Schwartz M, Fiel I, Thung S, Mazzaferro V, Bruix J, Bottinger E, Friedman S, Waxman S, Llovet JM: Genome-wide molecular profiles of HCV-induced dysplasia and hepatocellular carcinoma. Hepatology 2007;45:938-947.

8 Choi BI, Takayasu K, Han MC: Small hepatocellular carcinomas and associated nodular lesions of the liver: pathology, pathogenesis, and imaging findings. AJR Am J Roentgenol 1993;160:1177-1187.

9 Zech CJ, Reiser MF, Herrmann KA: Imaging of hepatocellular carcinoma by computed tomography and magnetic resonance imaging: state of the art. Dig Dis 2009;27:114-124.

10 Park YN: Update on precursor and early lesions of hepatocellular carcinomas. Arch Pathol Lab Med 2011;135: 704-715.

11 Sakamoto M, Hirohashi S, Shimosato Y: Early stages of multistep hepatocarcinogenesis: adenomatous hyperplasia and early hepatocellular carcinoma. Hum Pathol 1991;22:172-178.

12 Libbrecht L, Craninx M, Nevens F, Desmet V, Roskams T: Predictive value of liver cell dysplasia for development of hepatocellular carcinoma in patients with non-cirrhotic and cirrhotic chronic viral hepatitis. Histopathology 2001;39:66-73.

13 Plentz RR, Park YN, Lechel A, Kim H, Nellessen F, Langkopf BH, Wilkens L, Destro A, Fiamengo B, Manns MP, Roncalli M, Rudolph KL: Telomere shortening and inactivation of cell cycle checkpoints characterize human hepatocarcinogenesis. Hepatology 2007;45:968-976.

14 Borzio M, Bruno S, Roncalli M, Mels GC, Ramella G, Borzio F, Leandro G, Servida E, Podda M: Liver cell dysplasia is a major risk factor for hepatocellular carcinoma in cirrhosis: a prospective study. Gastroenterology 1995; 108:812-817.

15 Hytiroglou P, Park YN, Krinsky G, Theise ND: Hepatic precancerous lesions and small hepatocellular carcinoma. Gastroenterol Clin North Am 2007;36:867-887, vii.

16 The International Consensus Group for Hepatocellular Neoplasia: Pathologic diagnosis of early hepatocellular carcinoma: a report of the international consensus group for hepatocellular neoplasia. Hepatology 2009;49: 658-664.

17 Park YN, Kim MJ: Hepatocarcinogenesis: imaging-pathologic correlation. Abdom Imaging 2011;36:232-243.

18 Ferrell LD, Crawford JM, Dhillon AP, Scheuer PJ, Nakanuma Y: Proposal for standardized criteria for the diagnosis of benign, borderline, and malignant hepatocellular lesions arising in chronic advanced liver disease. Am J Surg Pathol 1993;17:1113-1123.

19 Desmet VJ: East-West pathology agreement on precancerous liver lesions and early hepatocellular carcinoma. Hepatology 2009;49:355-357.

20 International Working Party: Terminology of nodular hepatocellular lesions. Hepatology 1995;22:983-993.

21 Libbrecht L, Desmet V, Roskams T: Preneoplastic lesions in human hepatocarcinogenesis. Liver Int 2005; 25: 16-27.

22 Earls JP, Theise ND, Weinreb JC, DeCorato DR, Krinsky GA, Rofsky NM, Mizrachi H, Teperman LW: Dysplastic nodules and hepatocellular carcinoma: thin-section MR imaging of explanted cirrhotic livers with pathologic correlation. Radiology 1996;201:207-214.

23 Kobayashi M, Ikeda K, Hosaka T, Sezaki H, Someya T, Akuta N, Suzuki F, Suzuki Y, Saitoh S, Arase Y, Kumada $\mathrm{H}$ : Dysplastic nodules frequently develop into hepatocellular carcinoma in patients with chronic viral hepatitis and cirrhosis. Cancer 2006;106:636-647.

24 Hanna RF, Aguirre DA, Kased N, Emery SC, Peterson MR, Sirlin CB: Cirrhosis-associated hepatocellular nodules: correlation of histopathologic and MR imaging features. Radiographics 2008;28:747-769.

25 Sakamoto M, Hirohashi S, Shimosato Y: Early stages of multistep hepatocarcinogenesis: adenomatous hyperplasia and early hepatocellular carcinoma. Hum Pathol 1991;22:172-178.

26 Llovet JM, Chen Y, Wurmbach E, Roayaie S, Fiel MI, Schwartz M, Thung SN, Khitrov G, Zhang W, Villanueva A: A molecular signature to discriminate dysplastic nodules from early hepatocellular carcinoma in HCV cirrhosis. Gastroenterology 2006;131:1758-1767.

27 Lim JH, Kim MJ, Park CK, Kang SS, Lee WJ, Lim HK: Dysplastic nodules in liver cirrhosis: detection with triple phase helical dynamic CT. Br J Radiol 2004;77:911-916.

28 Khatri G, Merrick L, Miller FH: MR imaging of hepatocellular carcinoma. Magn Reson Imaging Clin North Am 2010;18:421-450, x.

29 Kojiro M, Roskams T: Early hepatocellular carcinoma and dysplastic nodules. Semin Liver Dis 2005;25:133142.

30 Park YN, Yang CP, Fernandez GJ, Cubukcu O, Thung SN, Theise ND: Neoangiogenesis and sinusoidal "capillarization" in dysplastic nodules of the liver. Am J Surg Pathol 1998;22:656-662.

31 Roncalli M, Roz E, Coggi G, Di Rocco MG, Bossi P, Minola E, Gambacorta M, Borzio M: The vascular profile of regenerative and dysplastic nodules of the cirrhotic liver: implications for diagnosis and classification. Hepatology 1999;30:1174-1178.

32 Park YN, Kim YB, Yang KM, Park C: Increased expression of vascular endothelial growth factor and angiogenesis in the early stage of multistep hepatocarcinogenesis. Arch Pathol Lab Med 2000;124:1061-1065. 
Park et al:: How to Differentiate Borderline Hepatic Nodules in Hepatocarcinogenesis: Emphasis on Imaging Diagnosis

33 Di Tommaso L, Franchi G, Park YN, Fiamengo B, Destro A, Morenghi E, Montorsi M, Torzilli G, Tommasini M, Terracciano L, Tornillo L, Vecchione R, Roncalli M: Diagnostic value of HSP70, glypican 3, and glutamine synthetase in hepatocellular nodules in cirrhosis. Hepatology 2007;45:725-734.

34 Di Tommaso L, Destro A, Seok JY, Balladore E, Terracciano L, Sangiovanni A, Iavarone M, Colombo M, Jang JJ Yu E, Jin SY, Morenghi E, Park YN, Roncalli M: The application of markers (HSP70 GPC3 and GS) in liver biopsies is useful for detection of hepatocellular carcinoma. J Hepatol 2009;50:746-754.

35 Ueda K, Matsui O, Kawamori Y, Nakanuma Y, Kadoya M, Yoshikawa J, Gabata T, Nonomura A, Takashima T: Hypervascular hepatocellular carcinoma: evaluation of hemodynamics with dynamic CT during hepatic arteriography. Radiology 1998;206:161-166.

36 Kitao A, Zen Y, Matsui O, Gabata T, Nakanuma Y: Hepatocarcinogenesis: multistep changes of drainage vessels at CT during arterial portography and hepatic arteriography-radiologic-pathologic correlation. Radiology 2009;252:605-614.

37 Takayama T, Makuuchi M, Hirohashi S, Sakamoto M, Okazaki N, Takayasu K, Kosuge T, Motoo Y, Yamazaki S, Hasegawa H: Malignant transformation of adenomatous hyperplasia to hepatocellular carcinoma. Lancet 1990;336:1150-1153.

38 Kutami R, Nakashima Y, Nakashima O, Shiota K, Kojiro M: Pathomorphologic study on the mechanism of fatty change in small hepatocellular carcinoma of humans. J Hepatol 2000;33:282-289.

39 Villanueva A, Newell P, Chiang DY, Friedman SL, Llovet JM: Genomics and signaling pathways in hepatocellular carcinoma. Semin Liver Dis 2007;27:55-76.

40 Zhang J, Krinsky GA: Iron-containing nodules of cirrhosis. NMR Biomed 2004;17:459-464.

41 Kitao A, Matsui O, Yoneda N, Kozaka K, Shinmura R, Koda W, Kobayashi S, Gabata T, Zen Y, Yamashita T, Kaneko S, Nakanuma Y: The uptake transporter OATP8 expression decreases during multistep hepatocarcinogenesis: correlation with gadoxetic acid enhanced MR imaging. Eur Radiol 2011;21:2056-2066.

42 Tsuboyama T, Onishi H, Kim T, Akita H, Hori M, Tatsumi M, Nakamoto A, Nagano H, Matsuura N, Wakasa K, Tomoda K: Hepatocellular carcinoma: hepatocyte-selective enhancement at gadoxetic acid-enhanced MR imaging - correlation with expression of sinusoidal and canalicular transporters and bile accumulation. Radiology 2010;255:824-833.

43 Lim JH and Choi BI: Dysplastic nodules in liver cirrhosis: imaging. Abdom Imaging 2002;27:117-128.

44 Matsui O, Kadoya M, Kameyama T, Yoshikawa J, Takashima T, Nakanuma Y, Unoura M, Kobayashi K, Izumi R, Ida $\mathrm{M}$, et al: Benign and malignant nodules in cirrhotic livers: distinction based on blood supply. Radiology 1991;178:493-497.

45 Piscaglia F, Bolondi L: Italian Society for Ultrasound in Medicine and Biology (SIUMB) Study Group on Ultrasound Contrast Agents: The safety of Sonovue in abdominal applications: retrospective analysis of 23188 investigations. Ultrasound Med Biol 2006;32:1369-1375.

46 Giorgio A, Calisti G, di Sarno A, Farella N, de Stefano G, Scognamiglio U, Giorgio V: Characterization of dysplastic nodules, early hepatocellular carcinoma and progressed hepatocellular carcinoma in cirrhosis with contrastenhanced ultrasound. Anticancer Res 2011;31:3977-3982.

47 Kudo M, Hatanaka K, Inoue T, Maekawa K: Depiction of portal supply in early hepatocellular carcinoma and dysplastic nodule: value of pure arterial ultrasound imaging in hepatocellular carcinoma. Oncology 2010; 78(suppl 1):60-67.

48 Jang HJ, Kim TK, Burns PN, Wilson SR: Enhancement patterns of hepatocellular carcinoma at contrastenhanced US: comparison with histologic differentiation. Radiology 2007;244:898-906.

49 Takahashi M, Maruyama H, Shimada T, Kamezaki H, Sekimoto T, Kanai F, Yokosuka O: Characterization of hepatic lesions $(</=30 \mathrm{~mm})$ with liver-specific contrast agents: a comparison between ultrasound and magnetic resonance imaging. Eur J Radiol 2013;82:75-84.

50 Sugimoto K, Moriyasu F, Saito K, Taira J, Saguchi T, Yoshimura N, Oshiro H, Imai Y, Shiraishi J: Comparison of Kupffer-phase Sonazoid-enhanced sonography and hepatobiliary-phase gadoxetic acid-enhanced magnetic resonance imaging of hepatocellular carcinoma and correlation with histologic grading. J Ultrasound Med 2012;31:529-538.

51 Ohama H, Imai Y, Nakashima O, Kogita S, Takamura M, Hori M, Seki Y, Sawai Y, Igura T, Fukuda K, Makino Y, Morimoto O, Ohsawa M, Sakamoto M, Murakami T: Images of Sonazoid-enhanced ultrasonography in multistep hepatocarcinogenesis: comparison with Gd-EOB-DTPA-enhanced MRI. J Gastroenterol 2014;49:1081-1093.

52 Omata M, Lesmana LA, Tateishi R, Chen PJ, Lin SM, Yoshida H, Kudo M, Lee JM, Choi BI, Poon RT, Shiina S, Cheng AL, Jia JD, Obi S, Han KH, Jafri W, Chow P, Lim SG, Chawla YK, Budihusodo U, Gani RA, Lesmana CR, Putranto TA, Liaw YF, Sarin SK: Asian Pacific Association for the Study of the Liver consensus recommendations on hepatocellular carcinoma. Hepatol Int 2010;4:439-474.

53 European Association for the Study of the Liver; European Organisation for Research and Treatment of Cancer: EASL-EORTC clinical practice guidelines: management of hepatocellular carcinoma. J Hepatol 2012; 56:908-943.

54 Mitchell DG, Bruix J, Sherman M, Sirlin CB: LI-RADS (Liver Imaging Reporting and Data System): summary, discussion, and consensus of the LI-RADS Management Working Group and future directions. Hepatology 2015;61:1056-1065

55 Honda H, Tajima T, Kajiyama K, Kuroiwa T, Yoshimitsu K, Irie H, Aibe H, Shimada M, Masuda K: Vascular changes in hepatocellular carcinoma: correlation of radiologic and pathologic findings. AJR Am J Roentgenol 1999;173:1213-1217. 
Park et al.: How to Differentiate Borderline Hepatic Nodules in Hepatocarcinogenesis:

56 Hayashi M, Matsui O, Ueda K, Kawamori Y, Gabata T, Kadoya M: Progression to hypervascular hepatocellular carcinoma: correlation with intranodular blood supply evaluated with CT during intraarterial injection of contrast material. Radiology 2002;225:143-149.

57 Yoon SH, Lee JM, So YH, Hong SH, Kim SJ, Han JK, Choi BI: Multiphasic MDCT enhancement pattern of hepatocellular carcinoma smaller than $3 \mathrm{~cm}$ in diameter: tumor size and cellular differentiation. AJR Am J Roentgenol 2009;193:W482-W489.

58 Kim SH, Lee WJ, Lim HK, Park CK: SPIO-enhanced MRI findings of well-differentiated hepatocellular carcinomas: correlation with MDCT findings. Korean J Radiol 2009;10:112-120.

59 Lee J, Lee WJ, Lim HK, Lim JH, Choi N, Park MH, Kim SW, Park CK: Early hepatocellular carcinoma: three-phase helical CT features of 16 patients. Korean J Radiol 2008;9:325-332.

60 Takayasu K, Furukawa H, Wakao F, Muramatsu Y, Abe H, Terauchi T, Winter TC 3rd, Sakamoto M, Hirohashi S: CT diagnosis of early hepatocellular carcinoma: sensitivity, findings, and CT-pathologic correlation. AJR Am J Roentgenol 1995;164:885-890.

61 Ito K: Hepatocellular carcinoma: conventional MRI findings including gadolinium-enhanced dynamic imaging. Eur J Radiol 2006;58:186-199.

62 Lee JM, Yoon JH, Kim KW: Diagnosis of hepatocellular carcinoma: newer radiological tools. Semin Oncol 2012; 39:399-409.

63 Bartolozzi C, Battaglia V, Bozzi E: HCC diagnosis with liver-specific MRI - close to histopathology. Dig Dis 2009; 27:125-130.

64 Van Beers BE, Pastor CM, Hussain HK: Primovist, Eovist: what to expect? J Hepatol 2012;57:421-429.

65 Taouli B, Koh DM: Diffusion-weighted MR imaging of the liver. Radiology 2010;254:47-66.

66 Burrel M, Llovet JM, Ayuso C, Iglesias C, Sala M, Miquel R, Caralt T, Ayuso JR, Sole M, Sanchez M, Bru C, Bruix J; Barcelona Clinic Liver Cancer Group: MRI angiography is superior to helical CT for detection of HCC prior to liver transplantation: an explant correlation. Hepatology 2003;38:1034-1042.

67 Rode A, Bancel B, Douek P, Chevallier M, Vilgrain V, Picaud G, Henry L, Berger F, Bizollon T, Gaudin JL, Ducerf C: Small nodule detection in cirrhotic livers: evaluation with US, spiral CT, and MRI and correlation with pathologic examination of explanted liver. J Comput Assist Tomogr 2001;25:327-336.

68 Martin J, Puig J, Darnell A, Donoso L: Magnetic resonance of focal liver lesions in hepatic cirrhosis and chronic hepatitis. Semin Ultrasound CT MR 2002;23:62-78.

69 Mathieu D, Paret M, Mahfouz AE, Caseiro-Alves F, Tran Van Nhieu J, Anglade MC, Rahmouni A, Vasile N: Hyperintense benign liver lesions on spin-echo T1-weighted MR images: pathologic correlations. Abdom Imaging 1997;22:410-417.

70 Muramatsu Y, Nawano S, Takayasu K, Moriyama N, Yamada T, Yamasaki S, Hirohashi S: Early hepatocellular carcinoma: MR imaging. Radiology 1991;181:209-213.

71 Kojiro M: Histopathology of liver cancers. Best Pract Res Clin Gastroenterol 2005;19:39-62.

72 Martin J, Sentis M, Zidan A, Donoso L, Puig J, Falco J, Bella R: Fatty metamorphosis of hepatocellular carcinoma: detection with chemical shift gradient-echo MR imaging. Radiology 1995;195:125-130.

73 Rimola J, Forner A, Tremosini S, Reig M, Vilana R, Bianchi L, Rodriguez-Lope C, Sole M, Ayuso C, Bruix J: Noninvasive diagnosis of hepatocellular carcinoma $</=2 \mathrm{~cm}$ in cirrhosis. Diagnostic accuracy assessing fat, capsule and signal intensity at dynamic MRI. J Hepatol 2012;56:1317-1323.

74 Park MS, Kim S, Patel J, Hajdu CH, Do RK, Mannelli L, Babb JS, Taouli B: Hepatocellular carcinoma: detection with diffusion-weighted versus contrast-enhanced magnetic resonance imaging in pretransplant patients. Hepatology 2012;56:140-148.

75 Park MJ, Kim YK, Lee MW, Lee WJ, Kim YS, Kim SH, Choi D, Rhim H: Small hepatocellular carcinomas: improved sensitivity by combining gadoxetic acid-enhanced and diffusion-weighted MR imaging patterns. Radiology 2012;264:761-770.

76 Kim YK, Kim CS, Han YM, Lee YH: Detection of liver malignancy with gadoxetic acid-enhanced MRI: is addition of diffusion-weighted MRI beneficial? Clin Radiol 2011;66:489-496.

77 Kim SH, Kim SH, Lee J, Kim MJ, Jeon YH, Park Y, Choi D, Lee WJ, Lim HK: Gadoxetic acid-enhanced MRI versus triple-phase MDCT for the preoperative detection of hepatocellular carcinoma. AJR Am J Roentgenol 2009; 192:1675-1681.

78 Sano K, Ichikawa T, Motosugi U, Sou H, Muhi AM, Matsuda M, Nakano M, Sakamoto M, Nakazawa T, Asakawa M, Fujii H, Kitamura T, Enomoto N, Araki T: Imaging study of early hepatocellular carcinoma: usefulness of gadoxetic acid-enhanced MR imaging. Radiology 2011;261:834-844.

79 Ichikawa T, Sano K, Morisaka H: Diagnosis of pathologically early HCC with EOB-MRI: experiences and current consensus. Liver Cancer 2014;3:97-107.

80 Rhee H, Kim MJ, Park YN, Choi JS, Kim KS: Gadoxetic acid-enhanced MRI findings of early hepatocellular carcinoma as defined by new histologic criteria. J Magn Reson Imaging 2012;35:393-398.

81 Kim MJ, Lee M, Choi JY, Park YN: Imaging features of small hepatocellular carcinomas with microvascular invasion on gadoxetic acid-enhanced MR imaging. Eur J Radiol 2012;81:2507-2512.

82 Kogita S, Imai Y, Okada M, Kim T, Onishi H, Takamura M, Fukuda K, Igura T, Sawai Y, Morimoto O, Hori M, Nagano H, Wakasa K, Hayashi N, Murakami T: Gd-EOB-DTPA-enhanced magnetic resonance images of hepatocellular carcinoma: correlation with histological grading and portal blood flow. Eur Radiol 2010;20:24052413. 
Park et al.: How to Differentiate Borderline Hepatic Nodules in Hepatocarcinogenesis: Emphasis on Imaging Diagnosis

83 Kudo M: Multistep human hepatocarcinogenesis: correlation of imaging with pathology. J Gastroenterol 2009 44(suppl 19):112-118.

84 Choi JY, Lee JM, Sirlin CB: CT and MR imaging diagnosis and staging of hepatocellular carcinoma. II. Extracellular agents, hepatobiliary agents, and ancillary imaging features. Radiology 2014;273:30-50.

85 Kitao A, Matsui O, Yoneda N, Kozaka K, Kobayashi S, Koda W, Gabata T, Yamashita T, Kaneko S, Nakanuma Y, Kita R, Arii S: Hypervascular hepatocellular carcinoma: correlation between biologic features and signal intensity on gadoxetic acid-enhanced MR images. Radiology 2012;265:780-789.

86 Kim YK, Lee WJ, Park MJ, Kim SH, Rhim H, Choi D: Hypovascular hypointense nodules on hepatobiliary phase gadoxetic acid-enhanced MR images in patients with cirrhosis: potential of DW imaging in predicting progression to hypervascular HCC. Radiology 2012;265:104-114.

87 Hyodo T, Murakami T, Imai Y, Okada M, Hori M, Kagawa Y, Kogita S, Kumano S, Kudo M, Mochizuki T: Hypovascular nodules in patients with chronic liver disease: risk factors for development of hypervascular hepatocellular carcinoma. Radiology 2013;266:480-490.

88 Yamamoto A, Ito K, Tamada T, Higaki A, Kanki A, Sato T, Tanimoto D: Newly developed hypervascular hepatocellular carcinoma during follow-up periods in patients with chronic liver disease: observation in serial gadoxetic acid-enhanced MRI. AJR Am J Roentgenol 2013;200:1254-1260. 